\title{
Efecto de la velocidad de enfriamiento sobre la trabajabilidad de la aleación AA2195
}

\section{Effect of Cooling Speed on the Workability of AA2195 Alloy}

\author{
Ernesto G. Maffia ${ }^{1 *} \quad$ Nicolás Hoffmann ${ }^{2} \quad$ Lucas E Feloy $^{1}$ \\ Juan L. Lacoste ${ }^{1} \quad$ Ana Laura Cozzarin ${ }^{1}$ \\ Recibido 26 de junio de 2019, aceptado 6 de julio de 2021 \\ Received: June 26, 2019 Accepted: July 6, 2021
}

\begin{abstract}
RESUMEN
Para disminuir el peso de los componentes utilizados en la industria aeroespacial se han desarrollado a nivel mundial aleaciones de aluminio de alta resistencia con litio como aleante principal. Este aleante reduce considerablemente el peso final de la aleación y además mejora las propiedades mecánicas al convertir a esta aleación en termotratable. Actualmente, estos materiales no se producen en el país y es difícil su importación ya que se trata de materiales estratégicos. El objetivo del presente trabajo es mostrar los resultados obtenidos en el estudio de los efectos del control de la velocidad de enfriamiento de una aleacion AA2195. El análisis y evaluación de los resultados se realizó a través de micrografías y ensayos de dureza. Se observa la aparición de estructuras de Widmanstätten de interfase incoherente al enfriar lentamente luego del recocido total, lo que hace inviable el conformado plástico posterior. Utilizando el temple luego del recocido, se inhibe la formación de estructuras de Widmanstätten, con lo que se mejora enormemente la trabajabilidad de la aleación.
\end{abstract}

Palabras claves: AA2195, Widmanstätten, velocidad de enfriamiento, trabajabilidad.

\begin{abstract}
High-strength aluminum alloys with lithium as the main alloy have been developed worldwide to reduce the weight of the components used in the aerospace industry. This addition considerably reduces the alloy's final weight and improves the mechanical properties by rendering it heat-treatable. Currently, these materials are not produced in the country, and it is difficult to import them because they are strategic materials. The present work aims to show the results obtained in studying the effects of cooling speed controlling the aforementioned material. The analysis and evaluation of the results were carried out through micrographs and hardness tests. The appearance of Widmanstätten structures with an inconsistent interface is observed when cooling slowly after total annealing, which makes subsequent plastic forming unfeasible. By using quench after annealing, the formation of Widmanstätten structures is inhibited, significantly improving the workability of the alloy.
\end{abstract}

Keywords: AA2195, Widmanstätten, cooling speed, plastic deformation, workability.

1 Universidad Nacional de La Plata. Departamento de Mecánica, Facultad de Ingeniería. La Plata, Argentina. Email: acozzarin@ing.unlp.edu.ar; lefeloy@gmail.com; juan.lacoste@ing.unlp.edu.ar; ernesto.maffia@ing.unlp.edu.ar

2 Universidad Nacional de La Plata. Instituto de Investigaciones Físicoquímicas Teóricas y Aplicadas, INIFTA. La Plata, Argentina. Email: inphoffmann@gmail.com

* Autor de correspondencia: ernesto.maffia@ing.unlp.edu.ar 


\section{INTRODUCCIÓN}

Las aleaciones de aluminio de alta resistencia son ampliamente utilizadas en la fabricación de aeronaves, misiles y en la industria aeroespacial, como así también, en accesorios y tanques para líquidos combustibles y de oxígeno. La razón de este uso radica en su alta resistencia específica (relación de resistencia mecánica a densidad), su buena resistencia a la corrosión y alta eficiencia de carga especialmente en diseños de compresión. $\mathrm{Al}$ ser un elemento de muy baja densidad $(0,53 \mathrm{~g} /$ $\mathrm{cm}^{3}$ ), ayuda a reducir considerablemente el peso de la aleación y permite aumentar el módulo de elasticidad del Aluminio en aproximadamente un $6 \%$ en cada adición de $1 \%$ en peso de Li [1, 2]. El Litio también eleva considerablemente la resistencia cuando se aplican tratamientos térmicos de endurecimiento por precipitación, ya que se crea la fase $\delta^{\prime}(\mathrm{Al} 3 \mathrm{Li})$, de características metaestable, con estructura ordenada y escaso desajuste con la matriz [3]. Al agregar $\mathrm{Cu}$, la aleación Al-Li tiende a aumentar el volumen de precipitación de la fase $\delta^{\prime}$ y la co-precipitación de las fases $\theta^{\prime}$ $(\mathrm{A} 12 \mathrm{Cu})$ y $\mathrm{T} 1$ (Al2CuLi). Los precipitados $\mathrm{T} 1$ son fundamentalmente las partículas de refuerzo en las aleaciones comerciales de Al-Li-Cu como la 2090. También puede haber la formación de otra fase, llamada T2 (A16CuLi3) que tiene características cuasi-cristalinas, y nuclea en los límites de los granos, reduciendo así la ductilidad y la dureza. Simulaciones computacionales [1] indican que la aleación, al ser enfriada en el equilibrio, produce una combinación de aluminio metálico con A15CuLi3, A12CuLi y A12CuMg. Las aleaciones de última generación reducen la cantidad de $\mathrm{Li}$ agregado en pos de superar las limitaciones en cuanto a de-laminación, anisotropía, resistencia a la fractura y soldabilidad, propiedades centrales para aplicaciones aeronáuticas [4].

La aleación AA2195 presenta como característica principal su gran soldabilidad, con una muy elevada respuesta frente al envejecimiento natural después de una etapa de deformación en frío.

Sin embargo, es importante reparar en que las velocidades de enfriamiento, después de aplicar diferentes tratamientos térmicos a una aleación de estructura compleja como la AA2195, pueden crear la posibilidad de aparición de segundas fases que dificulten a posteriori la capacidad del material de asimilar deformación y, consecuentemente, de limitar la viabilidad para obtener piezas sanas en todo su volumen y de buena terminación superficial. Comprender la influencia de la composición química y la microestructura permite, entonces, la optimización simultánea de la proporción de los aleantes y el procesado termo mecánico de la aleación.

En el presente trabajo se estudia el comportamiento de la aleación AA2195 en función de los tratamientos térmicos. Para ello se varían las condiciones del recocido total y los modos de enfriamiento. Se exponen los resultados a través del estudio microestructural y de la comparación de la trabajabilidad.

\section{MATERIALES Y MÉTODOS}

Los elementos aleantes utilizados para la obtención de la aleación AA2195 han sido incorporados en estado puro $(\mathrm{Cu}, \mathrm{Li}, \mathrm{Ag}, \mathrm{Zr}$ y $\mathrm{Mg})$. Debido a que tanto el $\mathrm{Mg}$ como el Li tienen un alto poder de oxidación durante la fusión de la aleación, estos se colocan en exceso al introducir la carga al crisol para compensar alguna posible pérdida de material en la oxidación y lograr que la composición química final continúe dentro de los rangos establecidos para la aleación AA2195. Por otro lado, al momento de incorporarlos aleantes al baño líquido, se utiliza una campana conjuntamente con la aplicación de una ráfaga continua de gas inerte sobre la superficie del metal fundido. Antes de realizar el vaciado de la aleación, esta es desgasificada con nitrógeno para disminuir la posible aparición de porosidad en la aleación vaciada.

Como se observa en la Figura 1, el llenado del molde es por surgencia. En este caso, se usa sólo un único canal de alimentación, ya que el espesor de la placa no requiere ataques adicionales y, además, el estrecho canal de ingreso del líquido, minimiza la influencia del último líquido caliente. Así mismo se coloca una mazarota de arena en la parte superior de la placa para minimizar la contracción superior en la placa.

La determinación de los elementos que forman la aleación fue realizada por un equipo portátil de fluorescencia de rayos X marca TITAN BRÜKER y sus resultados se presentan en la Tabla 1. 


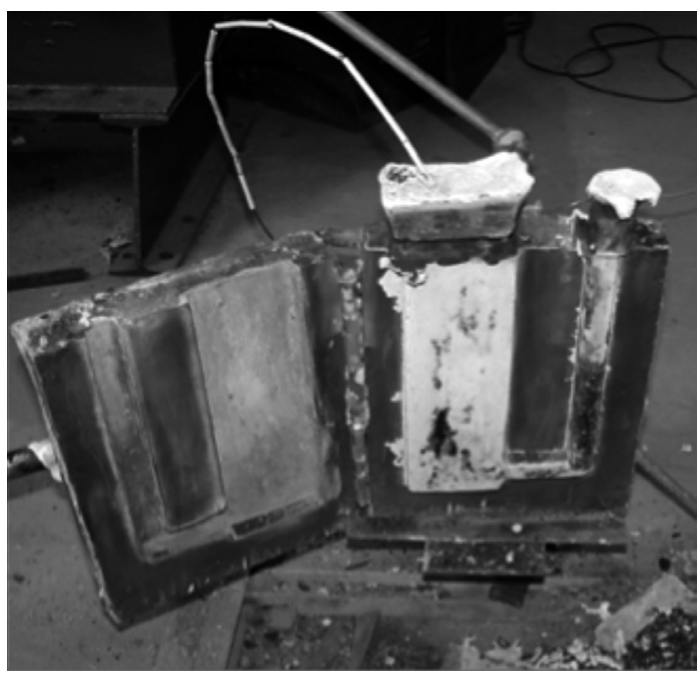

Figura 1. Molde metálico abierto con pieza fundida.

Tabla 1. Composición del metal vaciado.

\begin{tabular}{|l|c|c|}
\hline \multicolumn{1}{|c|}{ Elemento } & Muestra & AA2195 [5] \\
\hline Cobre $(\mathrm{Cu})$ & 4,2 & $3,7-4,3$ \\
\hline Litio $(\mathrm{Li})$ & 0,87 & $0,8-1,2$ \\
\hline Magnesio $(\mathrm{Mg})$ & 0,68 & $0,25-0,8$ \\
\hline Plata $(\mathrm{Ag})$ & 0,40 & $0,25-0,4$ \\
\hline Zirconio $(\mathrm{Zr})$ & 0,15 & $0,08-0,16$ \\
\hline
\end{tabular}

Antes de comenzar el estudio de la trabajabilidad de la aleación, esta se somete a un tratamiento térmico de homogeneizado a una temperatura de $480{ }^{\circ} \mathrm{C}$ por 20 horas para uniformizar la composición química. Luego se comienza a estudiar del comportamiento de la aleación, aplicando un tratamiento de recocido total; este tratamiento consiste en llevar el material a $420^{\circ} \mathrm{C}$ por 3 horas, seguido de un enfriamiento a una velocidad máxima de $30^{\circ} \mathrm{C} / \mathrm{h}$ hasta llegar a $290^{\circ} \mathrm{C}$. $\mathrm{Al}$ alcanzar este umbral de temperatura, o bien se deja que la aleación se enfrié en el horno, o se la templa en agua; este enfriamiento brusco tiene como objetivo detener la difusión de los elementos disueltos en solución sólida. Salvo que se indique lo contrario, todos los tratamientos térmicos son seguidos de enfriamiento al aire y se realizaron en un horno de resistencia eléctrica (Linberg) sin atmósfera de protección.

Se utiliza un laminador Duo (Lenis) de $25 \mathrm{~cm}$ de ancho de tabla, el cual tiene adosado un horno eléctrico de resistencia, en serie con los rodillos. Todas las muestras de este trabajo fueron caracterizadas por microscopia óptica (LEICA), utilizando siempre 200 aumentos. Todas las micrografías mostradas en este trabajo se obtuvieron a partir de preparación metalográfica convencional. Los reactivos utilizados fueron reactivo de Graff y reactivo de Keller [6].

Las mediciones de dureza se han realizado con un durómetro (Petri) usando la escala de dureza Brinell con penetrador de $5 \mathrm{~mm}$ y una carga de $250 \mathrm{~kg}$.

En el diagrama de flujo siguiente, se muestran las secuencias elegidas para estudiar el comportamiento del material en diferentes condiciones estructurales, destacándose en los recuadros naranjas la Figura 2, las estructuras logradas por los tratamientos termo mecánicos aplicados a la aleación AA2195.

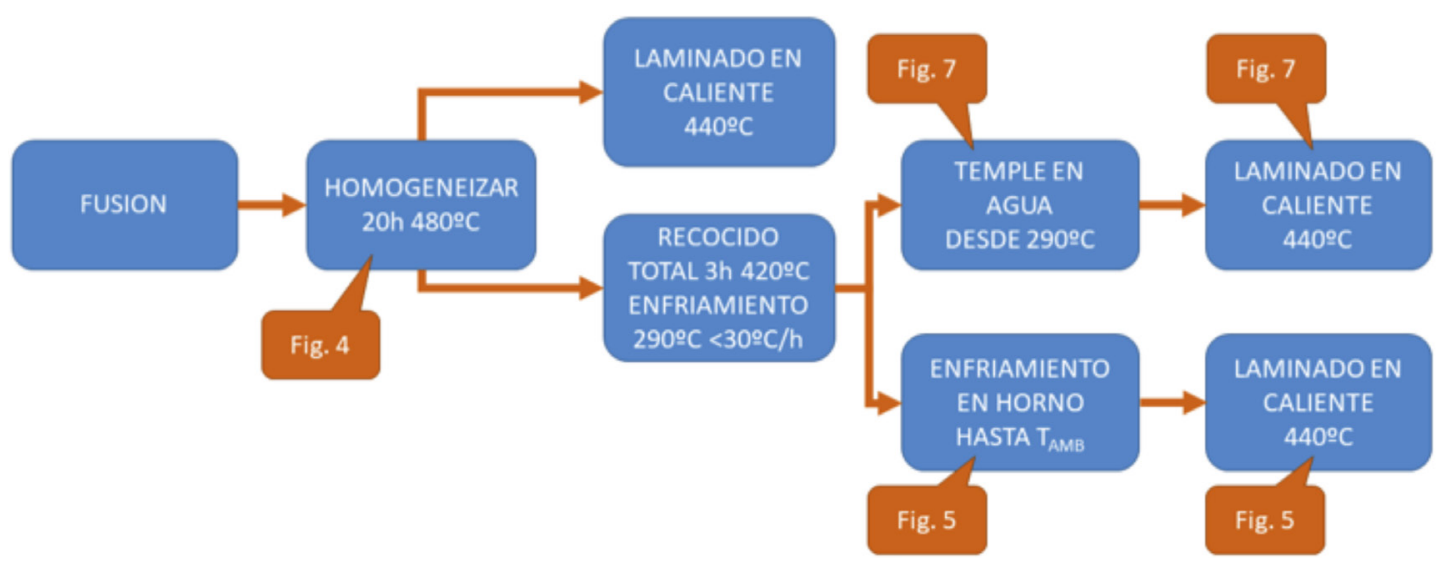

Figura 2. Diagrama del proceso realizado durante este trabajo. 


\section{RESULTADOS}

\section{Caracterización inicial al metal fundido}

Se generan muestras metalográficas inmediatamente al vaciado de la placa con el objetivo de relevar la condición microestructural inicial de la placa antes de comenzar con su procesado. Este estudio microestructural, además, permite evaluar la velocidad de solidificación observando el volumen de segregación en espacios interdendriticos.

En la Figura 3 se presentan los resultados obtenidos luego de realizar una metalografía. Se observa una microestructura de colada, con gran contenido de segregación interdendritica. Esta situación microestructural es nociva para el proceso de deformación plástica, por lo que debe ser minimizada o eliminada por algún tipo de tratamiento térmico. Para esto se debe utilizar la difusión a altas temperaturas, calentando el material hasta encontrar una región donde se forma una única fase.

\section{Condición de homogeneizado}

Se realiza un tratamiento térmico de homogeneizado para uniformizar la composición química del material e intentar disolver las fases segregadas durante el enfriamiento fuera de equilibrio. El resultado se presenta en la Figura 4 (izquierda), donde se observa una microestructura con menor segregación.

A continuación, para evaluar la trabajabilidad de la aleación en esta condición, se lamina en caliente hasta alcanzar una deformación de 11,5\% (Figura 4,

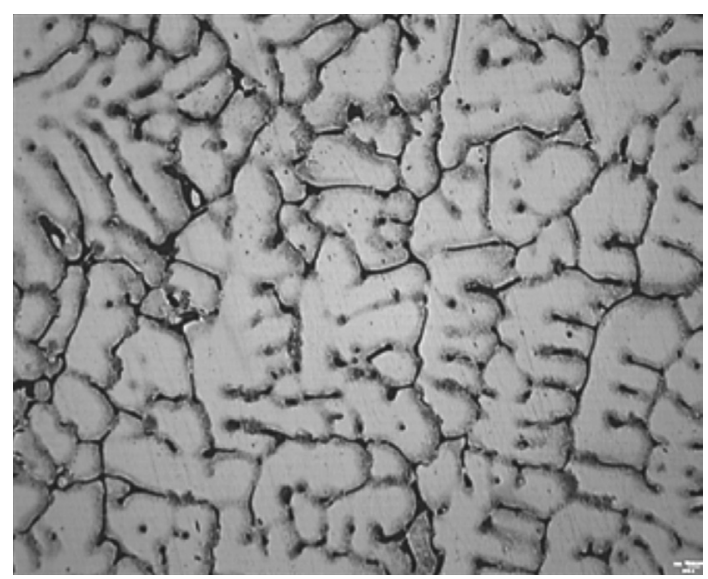

Figura 3. Muestra colada en molde metálico a temperatura ambiente (200x).

derecha). Puede observarse que la deformación no produce orientación preferencial ni grietas en los granos del material.

Nuevamente se lamina este material hasta un espesor de $25 \mathrm{~mm}$ (reducción aproximada de 17\%), hasta que se observa agrietamiento superficial de la placa donde suspende el laminado.

\section{Condición de Recocido Total}

Se realiza un recocido total de la aleación AA2195, con enfriamiento en horno. Los efectos del tratamiento térmico en la microestructura de la aleación, se analizan por microscopia óptica (Figura 5, izquierda), observándose estructuras
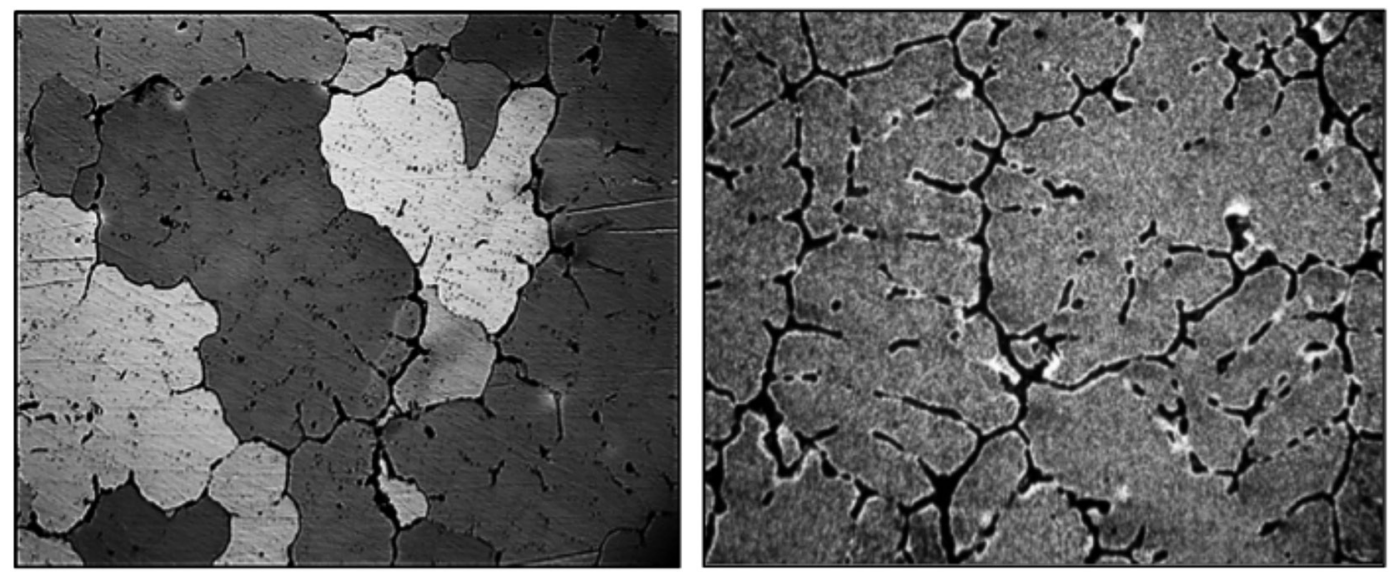

Figura 4. Izquierda: Material homogeneizado por $20 \mathrm{~h}$ a $480{ }^{\circ} \mathrm{C}$.Derecha: Mismo material tras laminarse en caliente un $11,5 \%$ a $440{ }^{\circ} \mathrm{C}$. (200x). 

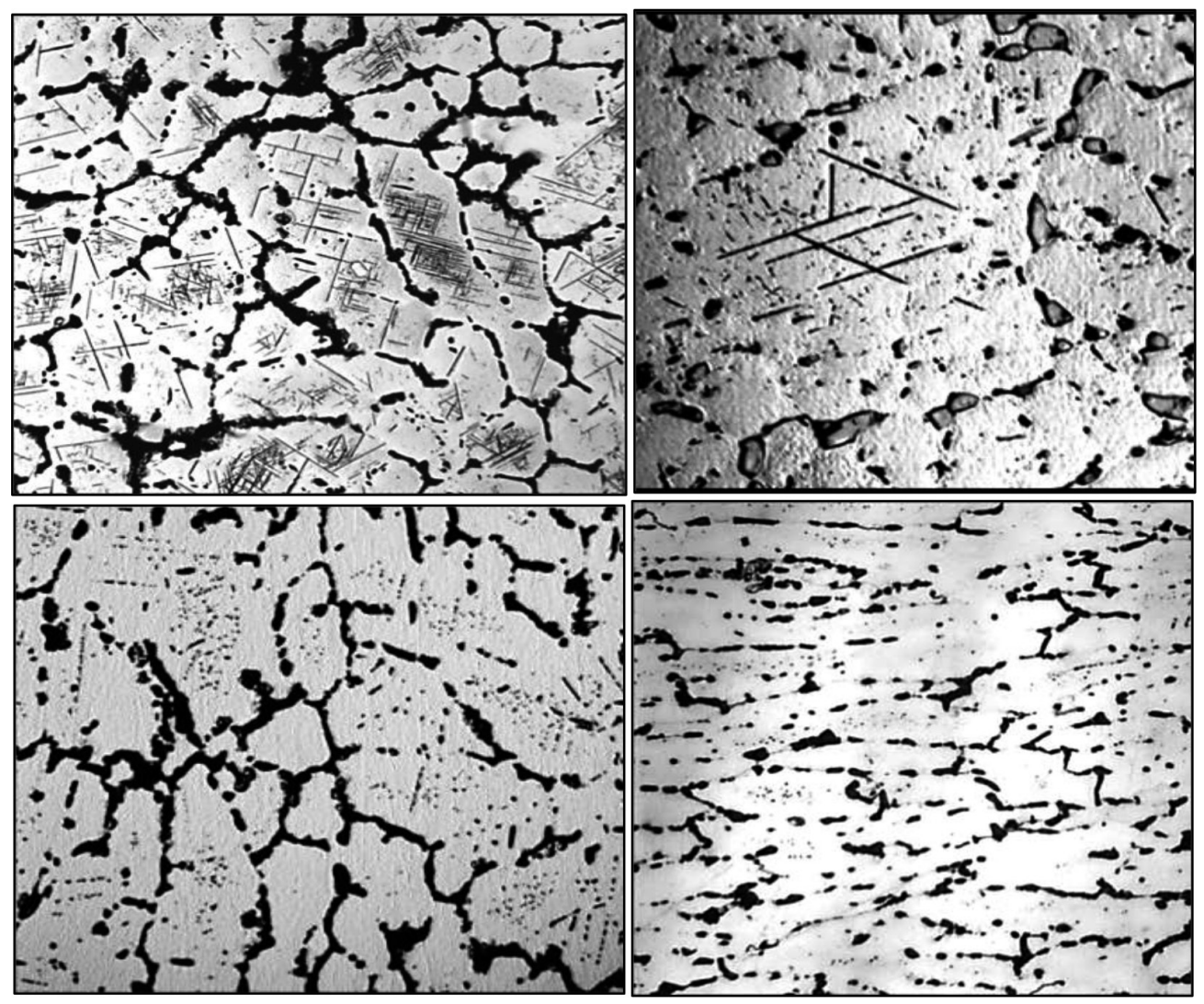

Figura 5. Material en condición de recocido total, con enfriamiento en horno. Superior izquierda: Sin deformación. Superior Derecha: Detalle a 800 aumentos de un grano donde se observan precipitados en borde de grano y la fase de Widmanstätten en el interior del mismo. Inferior Izquierda: 44\% de deformación en caliente, espesor final de 16,5 mm. Inferior Derecha: 55\% de deformación en caliente, espesor final de 13,5 mm. (200x).

de Widmanstätten dentro de los granos. Estas fases aciculares, tipo Widmanstätten se presentan formando ángulos de $90^{\circ}$ y $60^{\circ}$ entre ellas, lo cual es producto de la precipitación de la fase sobre los planos octaédricos de la estructura cúbica centrada en las caras de la fase madre [7]. Las mismas corresponden a una fase intermetálica incoherente de composición no determinada, informada por la bibliografía como fase T1 [8]. Esta fase nuclea preferencialmente en bordes de subgrano, donde debe crecer muy rápidamente durante el enfriamiento desde $290{ }^{\circ} \mathrm{C}$ a temperatura ambiente [9], a partir de esto puede suponerse que la fase en cuestión es $\mathrm{T} 1$, delimitando los bordes de subgrano.

La micrografía de la muestra deformada un $44 \%$ (Figura 5, inferior izquierda) apenas presenta los últimos restos de la estructura de Widmanstätten. Este resultado probablemente se deba a que el proceso de laminación se realiza a $440{ }^{\circ} \mathrm{C}$, donde se re disuelve parte de la misma, y al ser enfriada al aire, se inhibe la formación de la totalidad de las fases de Widmanstätten. Al 44\% de deformación (Figura 4, inferior izquierda) sigue sin poder visualizarse señales de orientación preferencial de la microestructura, evento que si es observable recién a los 55\% (Figura 5, inferior derecha). La micrografía presenta una reducción en la cantidad de segunda fase, probablemente porque el enfriamiento se realiza a velocidades fuera del equilibrio. A pesar de encontrarse fisuras superficiales en la placa laminada, se puede continuar laminando en caliente sin producir la rotura total del material. 
En la Figura 6 se presentan diagramas TTT (temperatura-tiempo-transformación) de la aleación AA2195. Analizando las trayectorias de enfriamiento de la aleación, se pueden preanunciar las fases que se encontrarían al final de las velocidades aplicadas. En el gráfico de la izquierda se observa la aparición de fases en la matriz, relacionados a los tratamientos térmicos realizados. La figura de la derecha corresponde a los bordes de subgrano. Evidentemente, la interpretación de estos modos de enfriamiento resulta en la ausencia de fases $\mathrm{T} 1$ para el enfriamiento por temple en agua (marcado con la flecha azul), donde no se permanece en temperaturas de alta velocidad de nucleación ni de crecimiento para dicha fase, mientras que el enfriamiento en horno hasta temperatura ambiente (en rojo, esquemático) atraviesa las temperaturas más adecuadas para la formación de fase T1. Para ambas fases, deberían encontrarse fases theta y theta' tanto en matriz como en borde de grano.

\section{Condición de Recocido Total y Temple}

Las fases de morfología acicular (también llamadas estructuras Widmanstätten) no favorecen la deformación plástica profunda durante el procesamiento de estos materiales, ya que las mismas se convierten en activos concentradores de tensiones [10, 11, 12]. Por esa causa, se estudia a la aleación AA2195 con tratamiento térmico de ablandamiento modificado. Esto significa que, tras alcanzar $290^{\circ} \mathrm{C}$ después del enfriamiento continuo, la aleación es templada. Esta acción tiene como objetivo solubilizar la fase acicular y así, influir el conformado posterior.

A continuación, en la Figura 7, se presenta una micrografía de una muestra atacada con Graff y Keller en la cual se percibe una disminución de la cantidad de fase dispuesta de manera interdendrítica y a su vez la fase acicular no se encuentra en el interior de los granos, por haber sufrido el temple, luego de alcanzar los $290{ }^{\circ} \mathrm{C}$. Esta condición estructural, sin dudas, es beneficiosa para el proceso de deformación plástica por laminación, pues se logran grandes deformaciones (>55\%) sin afectar la integridad de la chapa.

Como en los casos anteriores, se empieza a observar una orientación preferencial de los granos cuando la deformación alcanza el 55\%, como se muestra en la derecha de la Figura [7]. La superficie macroscópica de la placa presenta algunas grietas, pero en una cantidad menor al material en condición de recocido sin templado.

\section{Dureza}

Con la finalidad de conocer el efecto de los tratamientos térmicos aplicados en la aleacion

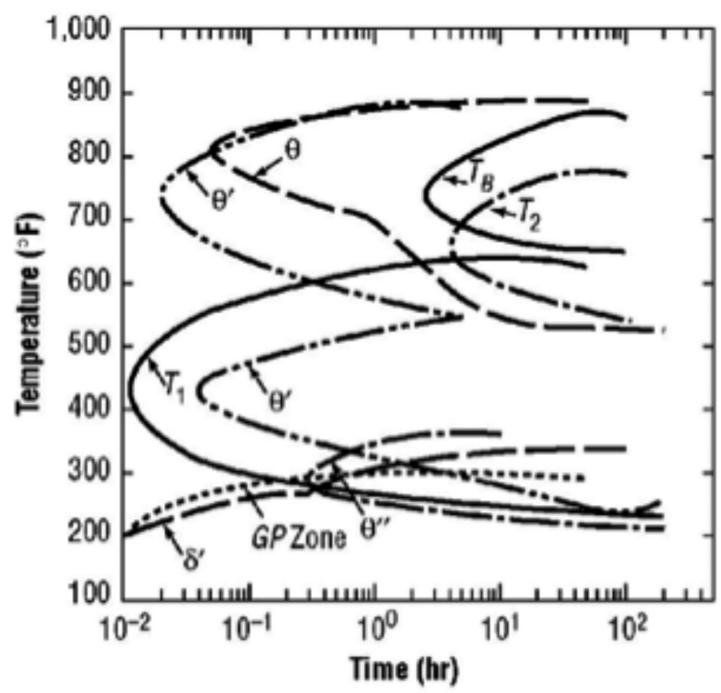

(a) Matrix

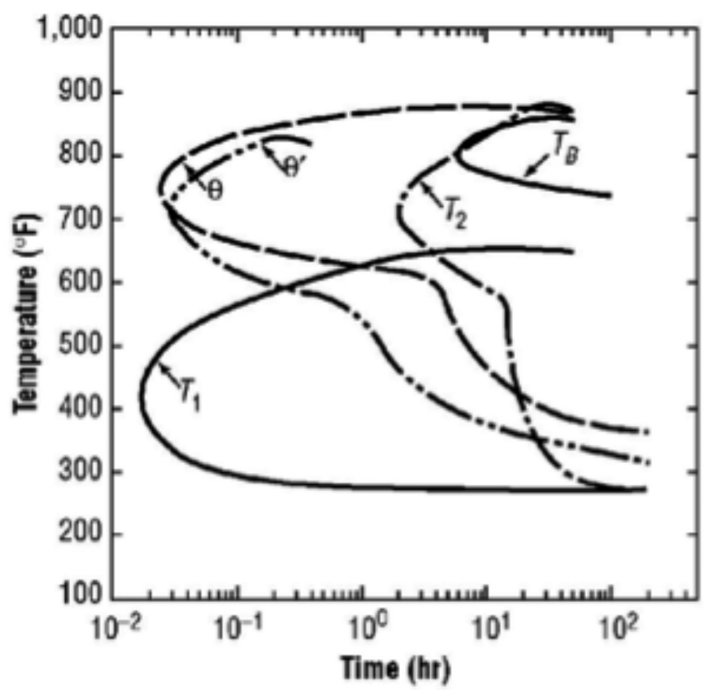

(b) Subgrain boundaries

Figura 6. Diagrama TTT de la aleación 2195. Se muestran los ciclos de enfriamiento aplicados en este trabajo: En rojo, enfriamiento en horno hasta temperatura ambiente por 24 horas, en azul, temple en agua al llegar a $290{ }^{\circ} \mathrm{C}$. [9]. 

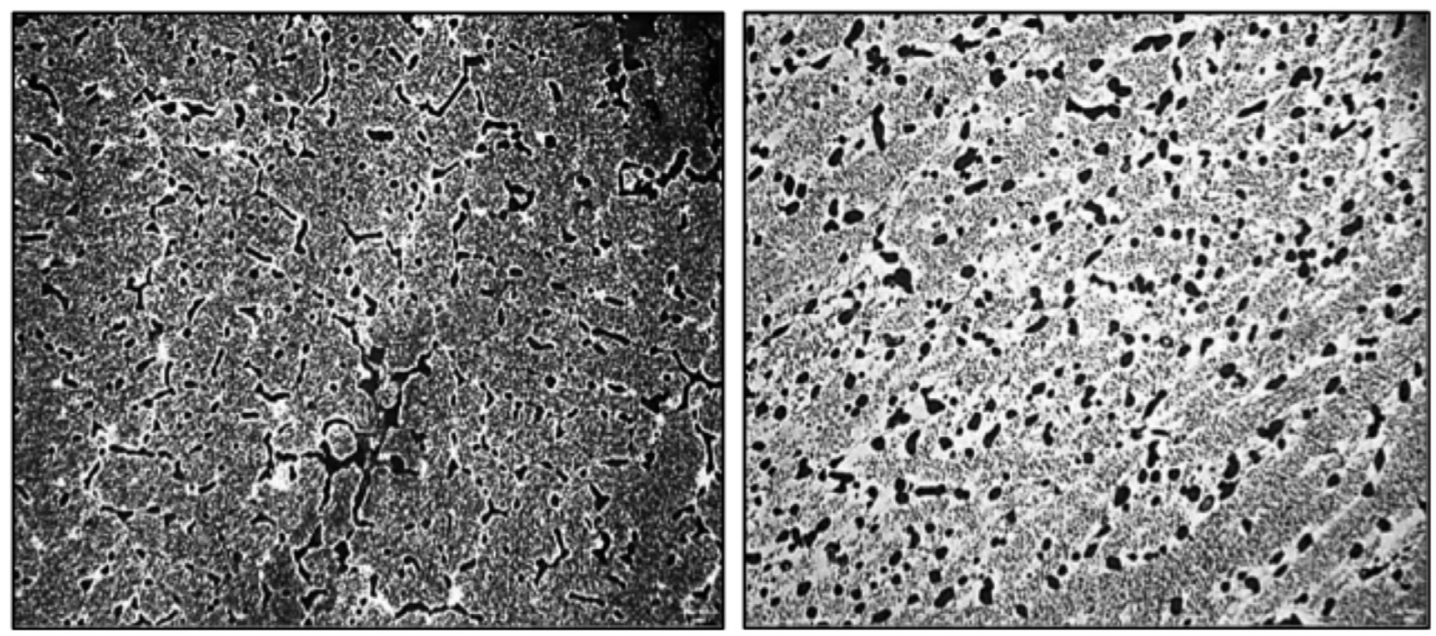

Figura 7. Izquierda: Material recocido y templado. Derecha: Deformado hasta reducción de espesor del 55\%. (200x).

AA2195 a lo largo de este estudio, se lleva a cabo un control permanente sobre las propiedades mecánicas del material, tomando como variable representativa el valor de dureza Brinell que exhibe cada una de las condiciones estudiadas. Los resultados se presentan en la Tabla 2.

A través del análisis de los diferentes valores de dureza, se observa el valor mayor de la aleacion en condición de colada. Este resultado es propio de una microestructura que se encuentra en un estado de máxima resistencia a la deformación, situación que coloca a la aleación AA2195 en una condición poco apropiada para cualquier proceso de conformado plástico.

El tratamiento de homogeneizado está pensado para distribuir los elementos químicos de la aleación y bajar la fragilidad. Sin embargo, al aplicarlo, no se logra disolver la totalidad de las segundas fases segregadas, lo cual se observa en las microestructuras y también en los datos de la tabla de durezas (la dureza apenas cae 11 puntos).

Se aplica, entonces, un recocido total con control de velocidad de enfriamiento. Los resultados de dureza confirman que la microestructura alcanza un estado de mínima resistencia a la deformación y por lo tanto se encuentra en mejores condiciones para afrontar el proceso de deformación plástica.

\section{CONCLUSIONES}

En el presente trabajo se estudian los efectos que produce el control de la velocidad de enfriamiento durante los tratamientos térmicos de la aleación AA2195. El análisis y evaluación de los resultados, permite extraer las siguientes conclusiones:

Tabla 2. Durezas (o resistencia a la deformación) de las diferentes condiciones microestructurales estudiadas en este trabajo.

\begin{tabular}{|l|c|}
\hline \multicolumn{1}{|c|}{ Condición de la Muestra } & Dureza [HB] \\
\hline De colada (As cast) & 96 \\
\hline Homogeneizado (20 hs a 480 C) & 85 \\
\hline Homogeneizado y luego un 11\% de laminación en caliente & 69 \\
\hline Recocido Total (3 hs a 420 C y enfriamiento en horno) & 73 \\
\hline Recocido Total modificado (3 hs a 420 C y enfriamiento en agua) & 58 \\
\hline Recocido Total modificado y luego un 86\% de laminación en Caliente (buena terminación superficial) & 75 \\
\hline
\end{tabular}


1. El análisis de los valores de dureza y de las micrografías de la estructura de colada, permiten observar una condición microestructural inapropiada para enfrentar un proceso de deformación plástica. Los ensayos de laminación de esta condición, fracasaron rápidamente.

2. El tratamiento de homogeneizado, sin embargo, no logra disolver la totalidad de las segundas fases que se forman durante la solidificación; la resistencia a la deformación de esta condición, apenas cambia respecto del material colado (Tabla 2).

3. Luego de aplicar un recocido total con enfriamiento lento a partir de $\operatorname{los} 290{ }^{\circ} \mathrm{C}$, aparecen en la microestructura fases aciculares; el laminado de la aleación AA2195 con estas características microestructurales, produce chapas con defectos superficiales.

4. Finalmente, se aplica un recocido total, pero con un enfriamiento rápido a partir de $\operatorname{los} 290^{\circ} \mathrm{C}$. Se observa que desaparecen de la microestructura las fases aciculares y se consigue una mejora en la terminación superficial.

Estos resultados permiten concluir que, si se inhibe la formación de estructuras aciculares, se mejora la trabajabilidad de la aleación.

\section{AGRADECIMIENTOS}

Los autores del siguiente trabajo agradecen a la UIDET PROINTEC I+D por haber facilitado el uso de sus instalaciones y proveer el material para este estudio.

\section{REFERENCIAS}

[1] A. Hekmat-Ardakam and E.M. Elgallad. "Microstructural evolution and mechanical properties of as - cast and T6-treated AA2195 DC cast alloy". Materials Science and Engineering: A. Vol. $558 \mathrm{~N}^{\mathrm{o}}$ 5, pp. 76-81. 2012. ISSN 0921-5093. DOI: 10.1016/j. msea.2012. 07.075.

[2] R.J. Rioja and J. Liu. "The Evolution of Al-Li Base Products for Aerospace and Space Applications". Metallurgical and Materials Transactions A.Vol. 44 No 9, pp. 3325-3337. 2012. ISSN impreso: 1073-5623. ISSN online: 1543-1940.DOI: 10.1007/s11661-012-1155-z.

[3] M. Tiryakioglu, J.T. Staley, G. Totten and D. Scott McKenzie. "Handbook of Aluminum:
Physical Metallurgy and the Effect of Alloying Additions". Marcel Dekker Inc. Vol. 1, pp. 152-159. New York, USA. ISBN: 0-8247-0494-0. 2003.

[4] Y. Xu, A. El-Aty, X. Guo, S. Zhang, Y. Ma and D. Chen. "Strengthening mechanisms, deformation behavior and anisotropic mechanical properties of Al-Li alloys: A review". Journal of Advanced Research. $\mathrm{N}^{\circ}$ 10, pp. 49-67. 2017. DOI: 10.1016/j. jare. 2017. 12.004.

[5] Material property data. "2195 aluminium composition spec". Fecha de consulta: 17 de abril de 2019. URL: http://www.matweb. $\mathrm{com} / \mathrm{search} /$ DataSheet.aspx? MatGUID= 4363dafc7f5545 68 8506d8b 4af1e9468

[6] ASM International Handbook Committee. "Metallography and Microstructures". ASM International. Vol. 9, pp. 1720. Ohio, USA. ISBN: 978-0-87170-706-2. 2004.

[7] G.A. Chadwick. "Metallography of phase transformations". Butterworth \& Co Publishers Ltd. ISBN-10: 0408703245. 1972.

[8] T. Dorin, A. Vahid and J. Lamb. "Chapter 11 - Aluminium lithium alloys". Fundamentals of Aluminium Metallurgy. Recent Advances. Woodhead Publishing Series in Metals and Surface Engineering, pp. 87-438. 2018. ISBN 9780081020630. DOI: 10.1016/B978-0-08102063-0.0 0011-4.

[9] P.S. Chen and B.N. Bhat. "Time-temperatureprecipitation behavior in ai-li alloy 2195". NASA Technical Report, NASA/TM--2002211548. 2002.

[10] R.L. Bodnar and S.S. Hansen. "Effects of Widmanstätten ferrite on the mechanical properties of a 0.2 pct C- 0.7 pct Mn steel". Metall Mater Trans A 25, pp. 763-773. 1994. DOI: $10.1007 /$ BF0266545

[11] G.Z. Koval'chuk, V.N. Geichenko, V.N. Yarmosh and L.V. Podobedova. "Effect of Widmanstatten ferrite on some properties of hypoeutectoid steel". Metal Science and Heat Treatment. Vol. 21 Issue 2, pp. 114-117. 1979. DOI: $10.1007 /$ BF00801483

[12] C. Xiaohui Huang, Ch. Voon Lim and S. Castagne. "Investigation of the workability and response of ti-6al-4v titanium alloys at lower elevated temperature and higher strain rate". 14th International ESAFORM Conference on Material Forming AIP Conf. Proc. 1353, pp. 1523-1528. 2011. DOI: 10.1063/1.3589733. 